\title{
Exploring the scope of Industrial Symbiosis: implications for practitioners
}

\author{
Maria Holgado, Dai Morgan, Steve Evans \\ Institute for Manufacturing, University of Cambridge, United Kingdom \\ mh769@cam.ac.uk; dcm32@cam.ac.uk; se321@cam.ac.uk
}

\begin{abstract}
Industrial Symbiosis can help improve the overall efficiency of the industrial system. The positive impact of implementing symbiotic exchanges between companies would benefit their host region through increased job creation and reduced environmental stress, whilst the entities engaged could benefit from a combination of additional revenue streams and reduced costs. However, in spite of the potential benefits of IS, there remains an implementation gap, with practitioners failing to fully exploit the possibilities of IS. The objective of this article is to provide a review of the current state of IS research in order to unlock current gaps of knowledge and practice, and identify research opportunities which will help close the implementation gap. The final aim is to explore and understand the areas practitioners willing to engage with IS need to consider in order to operationalize IS in their network.
\end{abstract}

Keywords Industrial Symbiosis, geographic proximity, manufacturing processes, energy efficiency, resource efficiency, network, Eco-Industrial Parks, waste management.

\section{Introduction}

Sustainability in manufacturing needs to be tackled from a holistic perspective [1]. Emerging as a construct drawn by observing and interpreting the behavior of industrial systems, Industrial Symbiosis (IS) can bring clear improvements at company level and also at network level. According WRAP [2] IS can help companies to reduce raw material consumption, carbon emissions and waste disposal costs while diverting waste from landfill and opening new business opportunities related to potential revenue streams from residues and by-products. IS can be considered a key element for the circular economy. Together with reuse, remanufacturing and recycling strategies, IS contributes to the creation of value through the exploitation of waste streams, emissions, and discarded products, in order to feed other products or production processes [3]. It also brings benefits from a social perspective. For example, at local and regional levels, IS can contribute to environmental improvements and new jobs creation [4].

Despite the claimed benefits of IS, its implementation remains challenging for practitioners implying a gap between theory and achievements in practice [5]. The 
objective of this article is to review concepts and dimensions associated with IS practice in order to unlock current gaps in knowledge and recognize research opportunities. The final aim is to understand which areas practitioners (i.e. any party who might be involved in the exploration or implementation of IS opportunities) need to consider and to explore how to develop support for those practitioners willing to engage with IS.

The next section introduces the emergence of IS as a concept, defining the scope of the inquiry. Subsequent sections concentrate on practical aspects identified from literature areas including IS, Industrial Ecology (IE) and Eco-Industrial Parks (EIP). We have searched the literature on the design, planning or implementation of IS for practitioner relevant insights. Key dimensions identified from the literature include: IS operationalization, geographic dispersion, network development and intermediaries. This is followed by a review of social aspects of IS. This article concludes with some final thoughts after a discussion on gaps and research opportunities related to the practical implementation of IS.

\section{Origins and definitions}

IS as an idea was inspired by the example of Kalundborg, in Denmark where a complex network of material, water and energy exchanges between industrial actors and the local municipality emerged over a period of around 40 years [6]. It was identified as an example of interest in the early stages of the Industrial Ecology movement, and became inspiration for the development of eco-industrial parks in the USA in the 1990's. A number of definitions are offered by the literature however there is no definitive definition.

Ehrenfeld and Gertler [7] reflect on IS as a focus on industrial efficiency at the system level, measured at the scale of the system as a whole, rather than at the factory level. Thus, some of the companies, viewed independently, may appear to be inefficient, yet environmental performance can be superior in the overall group of companies. In 2000, Chertow positioned IS as a part of the emerging field of Industrial Ecology which "demands resolute attention to the flow of materials and energy through local, regional and global economies". IS was described as "traditionally separate entities [engaged] in a collective approach to competitive advantage involving physical exchange of materials, energy, water"[8]. This description emphasized collaboration and geographical proximity as key factors in synergies, focusing, on the IE subset of flows operating at inter and intra firm levels.

Chertow later expanded on this view in 2007 [9], introducing the 3-2 heuristic ${ }^{1}$ to aid the identification of symbiotic examples in practice. Lombardi and Laybourn [10], drawing on the experience of a UK based scheme funded by the government from landfill tax, frame IS as a tool for innovative green growth stating that "IS engages diverse organizations in a network to foster eco-innovation and long-term culture

\footnotetext{
${ }^{1}$ i. e. that a minimum of 3 organizations (none of which is primarily involved in recycling as an industry) exchanging 2 resources as a minimum condition for symbiosis.
} 
change" and explicitly diminishing the emphasis on proximity as a key determinant of IS.

\section{Operationalizing Industrial Symbiosis}

What does it mean in practice? IS related exchanges can occur as a one-off material waste exchange(s) or more continuous flows can be exchanged between different entities with certain geographic proximity [8]. IS opportunities occur at the level of an industrial process [10] and can therefore be realized by a single company or factory (intra-firm IS) or in partnership with other companies (inter-firm IS).

How does it come about? IS can emerge unprompted from the interactions between companies as serendipitous arrangements. Some attempts to plan IS include the design of industrial estates and eco-industrial parks [11] [5]. Paquin and HowardGrenville [12] propose Facilitated IS as an intermediate arrangement between selforganized and planned IS. In facilitated IS symbiotic exchanges are enabled by a third party intermediary such as the National Industrial Symbiosis Programme (NISP) in UK which is the world's largest coordinating entity for by-product use between regional clusters [9][10]. Facilitation and coordination could also be seen as part of the evolution of IS after its initial establishment in order to enhance the potential opportunities for collaboration [13].

Company size. The mode of engagement with IS may strongly depend on company size. Larger companies with multiple sites are more likely to engage in intra-firm IS [14]. Conversely, SMEs would be more likely to need collaboration with others in order to realize IS opportunities. IS opportunities for SMEs could additionally come from "mutualisation", i.e. for sharing or creating new waste management infrastructures, facilities and services between them [15]. This type of solution would tackle the issue of "whether there is sufficient flow of materials to make IS worthwhile" [8].

\subsection{The geographical dispersion}

Geographic proximity between entities was identified as an enabler of the advantageous exchange of resources among different industries, however, certain types of waste may have trading opportunities at local, regional, national or global level [8]. Waste with high market value and relatively cheap transport cost such as metal, electrical and electronic equipment, plastics, paper and oil are mostly collected from and delivered to longer distances[16], thus, being candidates for IS applications outside the local scope. Some authors argue that the adequate scale for IS is the region [17] [18] whilst others emphasize local collaboration and partnership [19]). Diversity and complementarity of local organizations has been highlighted as an important factor to create high-value collaborations [20]. The local level therefore 
seems to provide an appropriate scale of application for certain types of IS, especially when considering the deployment of EIP that will bring a series of companies with common or complementary needs together to the same geographic location.

At a regional scale, there is some evidence that EIPs look beyond their local area, expanding the exchanges outside their own boundaries [5]. Zamorano et al. [21] consider proximity between industrial parks in a region as a positive aspect that enables collaboration on waste management processes and systems as reaching economies of scale. Similar findings are reported by Ruiz Puente et al. [15], whilst NISP first attempts to find resource matches at a regional level.

Chen et al. [16] suggest that exchanges already occurring at regional level do not imply the need to realize the exchanges at this level for all types of waste. At national level, evidence has been found of inter-regional exchanges facilitated by national programs; NISP coordinates different regions to facilitate matches where local or regional options are not available. National programs for IS applications and for the deployment of EIP have been funded in several countries so far: UK, US, Sweden, China, Japan, Germany, Spain, Italy, Korea [15] [20] [22] [23] [24] [25]. We have not found any intercountry application of IS in literature or practice so far.

\section{The network perspective}

The scope of IS network research to date focuses mainly on IS planning and design while coordination and management of IS networks are still little explored [13]. Cooperation is at the core of IS concept [10] [13]. IS itself implies a sense of cooperation and networking either when the resource exchange is done at factory/organization level or among different companies. Chertow's 3-2 heuristic [9] implies a network approach to IS, rather than a dyadic relationship between exchanging companies.

Inter-firm cooperation needs to be actively supported in order to keep the IS network running over time. An environment of trust can facilitate IS deployment, for example, reducing some related transaction costs: (i) search costs, related to the identification of opportunities for exchanges; (ii) negotiation costs, related to the agreement on the terms of the exchanges; (iii) enforcement costs, related to putting in effect the contract [13].

There are still few studies on IS network evolution and resilience over time. Some factors, such as the establishment of mutually beneficial transactions and a joint network vision during the planning process could assure long term commitment [26] [27] while others, such as the closure of any involved companies, an adverse reaction of local community or the global / national trends in particular sectors, can create disruption in the operation of the IS network and cause radical changes or even its decline [28]. Trade-offs regarding resilience and efficiency of IS networks have been studied. Resilience increases with the addition of different industries and redundant commodity exchanges [29] but decreases with interfirm dependency [30]. However, high-interfirm dependency increases the network's overall efficiency and reduces the risk of eco-efficiency losses. 


\subsection{The role of intermediaries}

Third parties have been often involved in recycling and selling the recycled materials. IS can bring more trading opportunities for them [8]. Waste-solution providers and specialized waste companies have played a key role during the NISP implementation [4] and the selection of authorized waste management companies in EIPs is seen as a means to reach economies of scale [21]. Although they can play a key role in IS networks, the study done by Posch [27] revealed that recycling companies tend to establish transaction-based dyadic relations and lack a shared network identity, network culture and shared objectives.

Occasionally, by-products cannot be used directly as inputs in manufacturing processes and require treatment by intermediaries or "middleman" [13]. These intermediaries need to be included in the network management and coordination processes, thus, having an impact on supply chain structures and complexity. Indeed, waste collectors and processors are envisaged to have a critical role in supply chains including IS exchanges [31]. Their capabilities increase the opportunities within a given supply chain or network, to create closed loops systems. Similarly, the waste management companies could expand their scope and develop more capabilities to support new exchanges [4], thus, creating more potential value for the whole IS network.

\section{The social aspects of Industrial Symbiosis}

While economic and environmental motives, methods and benefits are mostly present in IS literature, the social dimension of IS has been frequently neglected, being addressed by limited research or industrial applications [27] [32] [33]. However, attention is increasing on the social side of IS exchanges and network development. From a stakeholder theory perspective [34], there are numerous entities or groups that affect or are affected by the IS. Apart from the companies directly involved in the exchanges, other relevant stakeholders include industrialists, regulation bodies, interests groups and consumers [27] as well as local communities, regions or countries as a whole [4] [13] [35]. Additionally, intermediaries for waste management and treatment are also new stakeholders which will influence strongly the value obtained out of the symbiotic exchanges. Cities, towns or residential communities nearby industrial settings can also participate in IS exchanges. New symbiotic exchanges can be generated by linking the management of municipal solid waste (MSW) with local industries [16] [36].

The interactions among companies within IS networks are attracting more and more interest in the research community. Social factors included in IS studies relate to institutional capacity, culture change, inter-firm learning, social embeddedness and social capital. Institutional capacity regards the "recurring interactions between a group of actors that expands in number and range over time" [37] enabling the development of the IS network. The long-term thinking required for IS implementation will bring changes into companies' culture. IS can foster long-term culture change, environmental innovations at local scale and promote inter-firm 
learning and knowledge generation [10] [20] [27]. Thus, a highly cooperative organizational culture within the industrial area would be expected to contribute to a successful IS implementation [38]. In this regard, cross-sectorial innovations as well as new research and technology development were interesting outputs of NISP, enabled by mutual learning and firm-specific knowledge sharing among companies [10].

The role of trust has been emphasized as an enabler of IS but there is still little understanding on the mechanisms for building trust and cooperation [33]. This is being addressed by studies on social embeddedness and social capital. The concept of embeddedness has been adapted from the field of sociology to IS in order to understand how social and cultural aspects influence decision-making during IS planning and implementation [33]. Within an IS context, the social capital concept refers to network connections and relationships in the companies participating in the exchanges [39]. The social capital of middle managers can be crucial for IS opportunity identification and development, especially in self-organizing IS settings [40]. The study done by Doménech and Davies [33] connects emotional ties in IS relationships to IS project examination approach and the reciprocity in the IS network. The presence of emotional ties would influence IS project evaluation as a more heuristic approach and a global perspective are taken rather than narrow economic calculations and would, moreover, improve reciprocity between companies in the network as well as knowledge transfer and cooperation.

\section{Gaps and research opportunities for Industrial Symbiosis implementation}

Companies could see in IS an opportunity to extend their resource productivity [8] while transforming negative environmental externalities into positive environmental benefits [13]. However, practitioners need to be cautious as IS may not be the only or optimal mechanism to solve all energy and resource efficiency problems. IS opportunities should be compared to other possible improvement mechanisms in order to assess its viability and applicability and to find the most eco-efficient solution [20] [41]. For example, if disposal of waste is just a small percentage of operating costs or if there is not scarcity of resources in the area, IS may be less attractive [8]. The appropriateness of the solutions will be strongly influenced by contextual factors. These factors can be related to social, informational, technological, economic and political aspects that will conform a potentially enabling context for IS [42] as well as environmental factors that will influence the reliability and life span of the IS network [43]. There is then a need for further research on tools and methods that can support practitioners to identify, at early stages of ideation, the available opportunities for their waste streams and for their procurement activities from an IS viewpoint and to evaluate these opportunities against other possible strategies for efficiency improvements.

Government policies may influence a wide range of aspects when looking at IS networks [5] [22]. For example, waste management is still not a common concern for 
many top managers [27] and this may be inhibiting efforts devoted to IS development. Regulatory measures could support IS implementation and create higher awareness among companies' top management. Regulations that penalize lower waste hierarchy management levels and coordination programs to facilitate and assist companies during the IS opportunity identification stage are examples of possible positive government interventions [42]. Conversely, practice may be aided by the removal or amendment of legislation and regulation which inhibits IS, e.g. removing unnecessary bureaucracy and streamlining processes.

Thus, there is not a one-size-fits-all when planning and implementing IS as context specific characteristics will shape the scope and opportunities for IS in each individual case. These characteristics include but are not limited to; company size and production processes, geographical landscape and regional industrialization as well as country-specific trade regulations and policy. The high degree of characterization needed for the design of IS in different contexts means practitioners would benefit from support (e.g. tools and methods) developed specifically to address contextualization challenges for IS design and planning.

Last but not least, for IS to flourish in practice, all actors in a potential IS system need to derive value from the network. Understanding the benefits (both monetary and related to other forms of value) provided to all actors in the system will help create the levels of trust that will keep the IS system running. Especially in facilitated IS, once the facilitator has left the system, built trust and well-understood benefits could support the survival of the IS system. It appears therefore that reciprocity is a key principle for practitioners wishing to design and implement IS, with the reciprocal benefits of network participation potentially underpinning long term success of IS implementations.

\section{Concluding remarks}

IS was established as a term of art within the field of IE, capturing a particular configuration of industry, found in Kalundborg Denmark, and typified by a complex web of resource exchanges. The definitions offered for IS vary but the broad identity is a network of actors who exchange resources which would previously have been wasted in some way. This recovery of latent value can occur within companies, between companies who are often (but not always) proximate, and who may be from previously unrelated industries. Thus, a symbiotic relationship is established between processes (in terms of resources) and companies (in terms of value) contributing to competitive advantage.

Implementation and active design of IS systems however has proved challenging in practice, with numerous failed or partially successful exercises noted in the literature and few successors or equals to the original inspirational example identified. Where success has been achieved in practice and documented it has often emerged in incremental fashion, developing over time and contributing to the overall business or network goals. In a notable example (NISP), facilitation of knowledgeable experts 
and a capacity for innovation has been identified as key factors in encouraging resource based exchanges.

Drawing on the metaphor of IS, we can see that companies are already engaged in a complex web of exchanges of resources and value which is the basis of the product and service delivery system which we rely on. The notion of IS specifically refers to the waste which arises as a by-product of that system. From a waste hierarchy perspective we might choose to reduce or eliminate the wastes before recycling and re-using them. However the latent value which is contained within the wastes should also be considered and evaluated as part of the business proposition. Indeed when viewed through an innovation lens, we might re-conceive the framing of IS as part of the core business search for competitive advantage.

This suggests that IS is something which emerges from latent value associated with surplus resources within an industrial network, which is exploited through innovation and cooperation. The implication of this being that it is necessary to treat waste not as a problem whose negative effects are to be minimized but as a resource from which maximum value can be extracted.

Acknowledgements. This work was supported by the European Union's Horizon 2020 research and innovation program (grant $n^{\circ}$ 680570) and the EPSRC Centre for Innovative Manufacturing in Industrial Sustainability (grant $\mathrm{n}^{\circ} \mathrm{EP} / \mathrm{I} 033351 / 1$ ).

\section{References}

1. Tennant, M.: Sustainability and manufacturing. Future of Manufacturing Project, Evidence Paper 35. Foresight, Government Office for Science, London (2013)

2. WRAP.: What is industrial symbiosis. http://www.wrap.org.uk/content/what-industrialsymbiosis [Last accessed 19/11/2015], (2014)

3. Valkokari, K., Valkokari, P., Palomäki, K., Uusitalo, T., Reunanen, M., Macchi, M., Rana, P., Liyanage, J.P.: Road-mapping the business potential of sustainability within the European manufacturing industry. Foresight. 16(4), 360-384 (2014)

4. Paquin, R.L., Busch, T., Tilleman, S.G.: Creating economic and environmental value through industrial symbiosis. Long Range Planning. 48, 95-107 (2015)

5. Gibbs, D., Deutz, P.: Reflections on implementing industrial ecology through ecoindustrial park development. Journal of Cleaner Production. 15(17), 1683-1695 (2007)

6. Ehrenfeld, J.R.: Industrial ecology: a framework for product and process design. Journal of Cleaner Production. 5(1), 87-95 (1997)

7. Ehrenfeld, J., Gertler, N.: Industrial ecology in practice. The Evolution of Interdependence at Kalundborg. Journal of industrial Ecology, 1(1), 67-79 (1997)

8. Chertow, M. R.: Industrial symbiosis: literature and taxonomy. Annual review of energy and the environment. 25(1), 313-337 (2000)

9. Chertow, M..: Uncovering industrial symbiosis. Journal of Industrial Ecology. 11(1), 1130 (2007)

10. Lombardi, D. R., Laybourn, P.: Redefining industrial symbiosis. Journal of Industrial Ecology. 16(1), 28-37 (2012) 
11. Singhal, S., Kapur, A.: Industrial estate planning and management in India — an integrated approach towards industrial ecology. Journal of Environmental management. 66(1), 19-29 (2002)

12. Paquin, R., Howard-Grenville, J.: Facilitating regional industrial symbiosis: Network growth in the UK's National Industrial Symbiosis Programme. The social embeddedness of industrial ecology. 103-128. (2009)

13. Chertow, M., Ehrenfeld, J.: Organizing Self - Organizing Systems. Journal of Industrial Ecology. 16(1), 13-27 (2012)

14. Zhu, Q., Lowe, E.A., Barnes, D.: Industrial symbiosis in China: a case study of the Guitang Group. Journal of Industrial Ecology. 11(1),31-42 (2007)

15. Ruiz Puente, M.C., Romero Arozamena, E., Evans, S.: Industrial symbiosis opportunities for small and medium sized enterprises: preliminary study in the Besaya region (Cantabria, Northern Spain). Journal of Cleaner Production. 87, 357-374 (2015)

16. Chen, X., Fujita, T., Ohnishi, S., Fujii, M., Geng, Y..: The impact of scale, recycling boundary, and type of waste on symbiosis and recycling. Journal of Industrial Ecology. 16(1), 129-141 (2012)

17. Desrochers, P.: Industrial symbiosis: the case for market coordination. Journal of Cleaner Production. 12(8), 1099-1110 (2004)

18. Sterr, T., Ott, T.: The industrial region as a promising unit for eco-industrial development-reflections, practical experience and establishment of innovative instruments to support industrial ecology. Journal of Cleaner Production. 12(8), 947-965 (2004)

19. Heeres, R. R., Vermeulen, W.J.V., De Walle, F.B..: Eco-industrial park initiatives in the USA and the Netherlands: first lessons. Journal of Cleaner Production. 12, 8 985-995 (2004)

20. Mirata, M., Emtairah, T.: Industrial symbiosis networks and the contribution to environmental innovation: the case of the Landskrona industrial symbiosis programme. Journal of cleaner production. 13(10), 993-1002 (2005)

21. Zamorano, M., Grindlay, A., Molero, E., Rodríguez, M.I.: Diagnosis and proposals for waste management in industrial areas in the service sector: case study in the metropolitan area of Granada (Spain). Journal of Cleaner Production. 19(17), 1946-1955 (2011)

22. Mirata, M.: Experiences from early stages of a national industrial symbiosis programme in the UK: determinants and coordination challenges. Journal of Cleaner Production. 12(8), 967-983 (2004)

23. Park, H., Rene, E.R., Choi, S., Chiu, A.S.F.: Strategies for sustainable development of industrial park in Ulsan, South Korea-from spontaneous evolution to systematic expansion of industrial symbiosis. Journal of Environmental Management, 87(1), 1-13 (2008)

24. van Berkel, R.: Comparability of industrial symbioses. Journal of Industrial Ecology, 13(4), 483-486 (2009).

25. Bai, L., Qiao, Q., Yao, Y, Guo, J., Xie, M.: Insights on the development progress of National Demonstration eco-industrial parks in China. Journal of Cleaner Production. 70, 4-14 (2014)

26. Hiete, M., Ludwig, J., Schultmann, F.: Intercompany energy integration. Journal of Industrial Ecology. 16(5), 689-698 (2012) 
27. Posch, A.: Industrial recycling networks as starting points for broader sustainability oriented cooperation?. Journal of Industrial Ecology. 14(2), 242-257 (2010)

28. Mannino, I., Ninka, E., Turvani, M, Chertow, M.: The decline of eco-industrial development in Porto Marghera, Italy. Journal of Cleaner Production 100, 286-296 (2015)

29. Chopra, S. S., Khanna, V.: Understanding resilience in industrial symbiosis networks: Insights from network analysis. Journal of environmental management, 141, 86-94 (2014).

30. Zhu, J., Ruth, M.: Exploring the resilience of industrial ecosystems. Journal of environmental management. 122, 65-75 (2013)

31. Leigh, M., Li, X.: Industrial ecology, industrial symbiosis and supply chain environmental sustainability: a case study of a large UK distributor. Journal of Cleaner Production. 106 (15), 632-643 (2014)

32. Baas, L.: Industrial symbiosis in the Rotterdam Harbour and Industry Complex: reflections on the interconnection of the techno - sphere with the social system. Business Strategy and the Environment 17(5), 330-340(2008)

33. Doménech, T., Davies, M.: The role of embeddedness in industrial symbiosis networks: phases in the evolution of industrial symbiosis networks. Business Strategy and the Environment. 20(5), 281-296 (2011)

34. Freeman R.E.: Strategic management: A stakeholder approach. Pitman, Boston (1984)

35. Jensen, P.D., Basson, L, Hellawell, E.E., Leach, M.: 'Habitat' Suitability Index Mapping for Industrial Symbiosis Planning. Journal of Industrial Ecology. 16(1), 38-50 (2012)

36. Geng, Y., Tsuyoshi, F., Chen, X.: Evaluation of innovative municipal solid waste management through urban symbiosis: a case study of Kawasaki. Journal of Cleaner Production. 18(10), 993-1000 (2010)

37. Boons, F., Spekkink, W., Jiao, W.: A process perspective on industrial symbiosis. Journal of Industrial Ecology 18(3), 341-355 (2014)

38. Sakr, D., Baas, L., El-Haggar, S., Huisingh, D.: Critical success and limiting factors for eco-industrial parks: global trends and Egyptian context. Journal of Cleaner Production, 19(11), 1158-1169 (2011)

39. Ashton, W.S., Bain, A.C.: Assessing the "Short Mental Distance" in Eco - Industrial Networks. Journal of Industrial Ecology. 16(1), 70-82 (2012)

40. Jacobsen, N.B.: Industrial symbiosis in Kalundborg, Denmark: a quantitative assessment of economic and environmental aspects. Journal of Industrial Ecology. 10(1 - 2), 239-255 (2006)

41. Salmi, O.: Eco-efficiency and industrial symbiosis-a counterfactual analysis of a mining community. Journal of Cleaner Production. 15(17), 1696-1705 (2007)

42. Costa, I., Massard, G., Agarwal, A.: Waste management policies for industrial symbiosis development: case studies in European countries. Journal of Cleaner Production. 18(8), 815-822 (2010)

43. Romero, E., Ruiz, M.C.: Framework for Applying a Complex Adaptive System Approach to Model the Operation of Eco - Industrial Parks. Journal of Industrial Ecology. 17(5), 731-741 (2013) 